\title{
THE POISSON-LAGUERRE TRANSFORM
}

BY DEBORAH TEPPER HAIMO AND FRANK M. CHOLEWINSKI ${ }^{1}$

Communicated by N. Levinson, September 21, 1967

For $\alpha \geqq 0$, let $L_{n}^{\alpha}(x)$ denote the Laguerre polynomial of degree $n$ given by

$$
L_{n}^{\alpha}(x)=\left(x^{-\alpha} e^{x} / n !\right)(d / d x)^{n}\left(x^{n+\alpha} e^{-x}\right), \quad n=0,1, \cdots
$$

We define the Laguerre difference operator $\nabla_{n}$ by

$$
\nabla_{n} f(n)=(n+1) f(n+1)-(2 n+\alpha+1) f(n)+(n+\alpha) f(n-1) \text {. }
$$

Then the Laguerre difference heat equation is given by

$$
\nabla_{n} u(n, t)=\partial u(n, t) / \partial t \text {. }
$$

A Laguerre temperature is a solution $u(n, t)$ of $\left({ }^{*}\right)$ which is a $C^{1}$ function of $t$. The fundamental Laguerre temperature is the function $g(n ; t)=g(n, 0 ; t)$, where

$$
g(n, m ; t)=\int_{0}^{\infty} e^{-x t} L_{n}^{\alpha}(x) L_{m}^{\alpha}(x) d \Omega(x), \quad t>0,
$$

with

$$
d \Omega(x)=e^{-x} x^{\alpha} d x
$$

Corresponding to $g(n, m ; t)$ is its conjugate $g\left(n^{*}, m ; t\right)$ given by

$$
g\left(n^{*}, m ; t\right)=\int_{0}^{\infty} e^{-x t} L_{n}^{\alpha}(-x) L_{m}^{\alpha}(x) d \Omega(x), \quad t>0 .
$$

An important subclass of the class of Laguerre temperatures includes those Laguerre temperatures $u(n, t)$ which satisfy the condition

$u(n, t)=\sum_{m=0}^{\infty} g\left(n, m ; t-t^{\prime}\right) u\left(m, t^{\prime}\right) \rho(m), \quad \rho(m)=m ! / \Gamma(m+\alpha+1)$,

for every $t, t^{\prime}, 0<t^{\prime}<t$, with the series converging absolutely. Laguerre temperatures which belong to this subclass are said to have the Huygens property. The functions $g(n, m ; t)$ have this property.

1 The research of the first author was supported in part by the National Aeronautics and Space Administration under Grant NGR-14-008-009 and that of the second author by the National Science Foundation under Grant GP-7167. 
The Poisson-Laguerre transform of a function $\phi$ is formed with the kernel $g(n, m ; t)$ and is given by

$$
u(n, t)=\sum_{m=0}^{\infty} g(n, m ; t) \phi(m) \rho(m),
$$

whenever the series converges. Within its regions of convergence, the Poisson-Laguerre transform is a Laguerre temperature.

The object of this paper is to summarize the principal results derived in the development of an inversion and representation theory for the Poisson-Laguerre transform. Details and proofs will appear in $[1]$.

Basic to the development of the theory are the properties of the functions $g(n, m ; t), g\left(n^{*}, m ; t\right)$, and quotients of these. For example, the fact that, for $0<t<t_{0} \leqq 1, g(n, m ; t) / g\left(n_{0}, m ; t_{0}\right)$ is a positive monotone decreasing function of $m$ for large $m$ is a determining factor in the convergence behavior of the Poisson-Laguerre transform given in the following result.

THEOREM 1. If $u(n, t)=\sum_{m=0}^{\infty} g(n, m ; t) \phi(n) \rho(m)$ converges conditionally for $\left(n_{0}, t_{0}\right), n_{0}$ a nonnegative integer, $0<t_{0} \leqq 1$, then it converges for all $n=0,1, \cdots$, and $t, 0<t \leqq t_{0} \leqq 1$.

Further, the boundedness of $g\left(n^{*}, m ; t\right) / g\left(n^{*}, m ; t_{0}\right)$ as a function of $m$ enables us to establish the following convergence for the conjugate transform.

THEOREM 2. If $u(n, t)=\sum_{m=0}^{\infty} g(n, m ; t) \phi(m) \rho(m)$ converges absolutely for $t=t_{0}, 0<t_{0} \leqq 1$, then so does

$$
u\left(n^{*}, t\right)=\sum_{m=0}^{\infty} g\left(n^{*}, m ; t\right) \phi(m) \rho(m),
$$

for $0<t \leqq t_{0} \leqq 1$.

Similar considerations are needed to develop the following fundamental inversion result.

TheOREM 3. Let $\sum_{m=0}^{\infty} g(n, m ; t) \phi(m) \rho(m)$ converge for $\left(n_{0}, t_{0}\right), n_{0} a$ nonnegative integer, $0<t_{0} \leqq 1$. Then

$$
\lim _{t \rightarrow 0^{+}} \sum_{m=0}^{\infty} g(n, m ; t) \phi(m) \rho(m)=\phi(n) .
$$

We have, in addition, a conjugate inversion formula. 
TheOREM 4. Let $u(n, t)=\sum_{m=0}^{\infty} g(n, m ; t) \phi(m) \rho(m)$ converge absolutely for $\left(n_{0}, t_{0}\right), n_{0}$ a nonnegative integer, $0<t_{0} \leqq 1$. Then

$$
\phi(n)=\sum_{m=0}^{\infty} g\left(n^{*}, m ; t\right) u\left(m^{*}, t\right) \rho(m),
$$

for $0<t \leqq t_{0} \leqq 1$.

The fact that a nonnegative Laguerre temperature has the Huygens property plays a central role in the characterization of Laguerre temperatures which can be represented by Poisson-Laguerre transforms of nonnegative functions. We have the following representation theorem.

THEOREM 5. A necessary and sufficient condition that

$$
u(n, t)=\sum_{m=0}^{\infty} g(n, m ; t) \phi(m) \rho(m)
$$

with $\phi(m)$ nonnegative and the series converging for $n=0,1, \cdots$, $0<t<c$ is that $u(n, t)$ be a nonnegative Laguerre temperature there.

\section{REFERENCES}

1. F. M. Cholewinski and D. T. Haimo, Laguerre temperatures, Proc. Conf. on New Directions in Orthogonal Expansions and their Continuous Analogues, Southern Illinois University Press, 1968.

2. I. I. Hirschman, Jr., Laguerre transforms, Duke Math. J. 30 (1963), 495-510.

SOUTHERN ILlinois UNIVERSITY, EDWARDSVILle, AND

Clemson University 